\title{
Philosophiques
}

\section{Bernard-Henry Levy : le mal radical ou la philosophie du désespoir}

\section{André Paradis}

Volume 10, numéro 1, avril 1983

URI : https://id.erudit.org/iderudit/203210ar

DOI : https://doi.org/10.7202/203210ar

Aller au sommaire du numéro

Éditeur(s)

Société de philosophie du Québec

ISSN

0316-2923 (imprimé)

1492-1391 (numérique)

Découvrir la revue

Citer cet article

Paradis, A. (1983). Bernard-Henry Levy : le mal radical ou la philosophie du désespoir. Philosophiques, 10(1), 3-14. https://doi.org/10.7202/203210ar
Résumé de l'article

La Barbarie à visage humain de Bernard-Henri Lévy traduit le désenchantement d'une génération de jeunes intellectuels français aussi bien face au marxisme, à son enseignement théorique, à ses crédos politiques, à ses prétentions de révolutionner les rapports sociaux que face au capitalisme, fut-il répressivement tentaculaire et « rationnel » ou tout simplement énergumène. En rupture de ban avec ses " doctes maîtres ", Althusser, Poulantzas, Deleuze ou Lyotard, Lévy pose que toute action politique militante, de gauche ou de droite, conduit irrémédiablement, par delà ses mots d'ordre de libération et de progrès, à la reproduction du Prince, du Pouvoir, tout pouvoir par essence tendant à l'absolu et au totalitarisme. La Maîtrise est la loi de ce monde et toute théorie socio-politique, tout projet de société ne peuvent être que des masques truculents de promesses dont il faut bien s'affubler pour que s'opère dans l'espoir la circularité répétitive (ré-volution) de cette Maîtrise. L'histoire à cet égard ne fait que tourner en rond et notre siècle n'est que l'ultime achèvement en extension, mais à " visage " humain cette fois, d'une barbarie plusieurs fois millénaire. Que reste-t-il au nouveau philosophe sinon de proclamer à tout jamais le divorce de l'Éthique et de la Politique, de la candeur et de l'imposture, du fond de sa retraite solipsiste et libertaire?
Ce document est protégé par la loi sur le droit d'auteur. L'utilisation des services d’Érudit (y compris la reproduction) est assujettie à sa politique d'utilisation que vous pouvez consulter en ligne.

https://apropos.erudit.org/fr/usagers/politique-dutilisation/ 


\title{
ARTICLES
}

\section{BERNARD-HENRI LEVY : LE MAL RADICAL OU LA PHILOSOPHIE DU DÉSESPOIR}

\author{
par André Paradis
}

RÉSUMÉ. La Barbarie à visage humain de Bernard-Henri Lévy traduit le désenchantement d'une génération de jeunes intellectuels français aussi bien face au marxisme, à son enseignement théorique, à ses crédos politiques, à ses prétentions de révolutionner les rapports sociaux que face au capitalisme, fut-il répressivement tentaculaire et " rationnel » ou tout simplement énergumène. En rupture de ban avec ses "doctes maîtres ", Althusser, Poulantzas, Deleuze ou Lyotard, Lévy pose que toute action politique militante, de gauche ou de droite, conduit irrémédiablement, par delà ses mots d'ordre de libération et de progrès, à la reproduction du Prince, du Pouvoir, tout pouvoir par essence tendant à l'absolu et au totalitarisme. La Maîtrise est la loi de ce monde et toute théorie socio-politique, tout projet de société ne peuvent être que des masques truculents de promesses dont il faut bien s'affubler pour que s'opère dans l'espoir la circularité répétitive (ré-volution) de cette Maîtrise. L'histoire à cet égard ne fait que tourner en rond et notre siècle n'est que l'ultime achèvement en extension, mais à "visage " humain cette fois, d'une barbarie plusieurs fois millénaire. Que reste-t-il au nouveau philosophe sinon de proclamer à tout jamais le divorce de l'Éthique et de la Politique, de la candeur et de l'imposture, du fond de sa retraite solipsiste et libertaire?

Que le nouveau philosophe ait pu conclure que notre siècle est un siècle de barbarie, barbarie du Capital tentaculaire et du fascisme, barbarie technicienne et bureaucratique, barbarie des savants maîtres de la parole et du discours, barbarie de la pla-

1. Cf. La Barbarie à visage bumain. Coll. «Figures ». Paris. Grasset, 1977. 
nification et du progrès, barbarie de l'idéologie autorisée et de l'homogène, que le nouveau philosophe ait pu conclure aussi que l'essence du socialisme c'est le stalinisme, que le socialisme n'est et ne peut être pas essence que le Goulag et les camps concentrationnaires, que le socialisme, outre le fait d'être une imposture, "un mensonge qui fait vivre " ${ }^{2}$, n'est qu'une modalité obligée du Capital, tout cela n'a pas de quoi surprendre.

Car pour cette philosophie que l'on dit nouvelle et qui nous reconduit en fait en cœur même du nihilisme et de la pensée libertaire, la barbarie n'est rien d'autre que l'expression d'une nécessité. "La barbarie, dit le nouveau philosophe, était à l'origine et c'est l'origine qui se déploie " ${ }^{3}$ dans cette civilisation du progrès où progresser c'est avancer mais avancer dans le déclin sans fin qui marque l'agonie prolongée de l'Occidént.

Et s'il en est ainsi, s'il s'agit là d'une fatalité, c'est que par delà le fait brut et empirique de l'histoire, il y a une ontologie qui commande l'histoire, c'est que par delà les sociétés humaines il y a une ontologie de l'homme qui, tel un sort maléfique jeté sur le troupeau humain, rend risible toute rébellion et naif tout projet de société.

Qu'est-ce donc que le monde ? demandera-t-on en effet au nouveau philosophe dans l'espoir de récolter le juste sarcasme que mérite la platitude de la question ainsi posée. La réponse pourtant ne tarde pas :

"Le monde est un désastre dont l'homme est le sommet " ${ }^{4}$.

Qu'est-ce alors que l'homme? demandera-t-on.

"L'homme n'est jamais qu'un Dieu manqué et une espèce de raté $"^{5}$.

Le mal est profond, il est à la racine des choses, il est radical. Le malheur vient en effet de ce que l'homme soit pétri de socialité, qu'il ne puisse subsister et être autrement que par

\footnotetext{
2. Op. cit., p. 97.

3. Idem, cf. p. 136.

4. Idem, p. 85

5. Idem, p. 85 .
} 
"l'intarissable horreur du lien social " ${ }^{6}$. «Il y a sûrement dans le fait même des sociétés, dit Bernard-Henri Lévy, quelque chose qui voue les hommes à la servitude et au malheur " $^{7}$. "Il y a sans doute quelque chose dans le pur fait de se rassembler qui rend le Maître nécessaire, inévitable, incontournable ${ }^{8}$. Et «le malheur durera tant que la nature humaine se coalisera en lien social $»$.

S'il y a donc histoire, ce ne peut être finalement pour cette nouvelle ontologie du mal de vivre que celle d'un cycle infernal et toujours identique à lui-même qui reconduit sans cesse l'homme au néant de sa servitude initiale, celle du lien social, celle à laquelle on ne peut échapper, celle qui rend compte de toutes les autres servitudes humaines.

La fatalité est telle en conséquence que "l'idée d'une société bonne est un rêve absurde, une contradiction dans les termes ${ }^{10}$. "L'idée d'un bien public est une idée de rêveurs, vite mués en assassins ${ }^{11}$. Toute société ne peut être que barbare puisque la socialité originelle de l'homme rend inévitable l'aménagement de l'État, que l'État est pouvoir et que tout pouvoir tend à l'absolu ${ }^{12}$. Notre siècle est le point culminant de ce procès d'universalisation du pouvoir qui rejoint l'individu jusque dans ses repères les plus secrets.

L'homme est donc menotté aujourd'hui plus qu'il ne l'était encore à l'origine. Toute révolte est vaine puisqu'elle se mue de façon incessante en un nouveau mode de servitude.

Toute recherche d'individuation est illusoire puisque l'individu n'est et ne peut être que le double patenté de l'État et qu' " il n'y a pas d'individualisme qui ne porte en lui le germe et la promesse d'une forme de totalitarisme ${ }^{13}$.

6. Idem, p. 36 .

7. Idem. p. 35.

8. Idem, p. 35 .

9. Idem, p. 39.

10. Idem, p. 53.

11. Idem. p. 38.

12. Idem, cf. p. 156.

13. Idem. p. 81 . 
Toute dissidence est imaginaire puisqu' « il n'y a pas de dissidence qui ne se réduise bien vite à une grimace de l'homogène $"{ }^{14}$.

Toute libération de la parole est un leurre car « s'il y a parole c'est qu'il y a socialité et que la socialité c'est la guerre ${ }^{15}$.

La Maîtrise est donc la loi de ce monde ; le pouvoir c'est le tout du réel et du monde ${ }^{16}$.

Voilà donc l'étrange tableau de l'humanité et de l'histoire que nous brosse le nouveau philosophe et c'est au nom de cette ontologie du mal radical et du désespoir, c'est au nom d'une lucidité qui se veut radicale elle aussi, sans détour et sans fard, que le nouveau philosophe, ce prophète de malheur, pourfend tous les chevaliers de l'optimisme, tous complices de cette gigantesque imposture qu'est celle du progrès et du sens de l'histoire :

Les technocrates modernistes, les sociologues du travail, les prophètes de l'âge post-industriel, les apôtres des classes moyennes, mais surtout, oui surtout les socialistes car le socialisme dit-on, a tout l'apparat d'une nouvelle religion, et ses militants ont toutes les propriétés du sacerdoce laïc, « animés » qu'ils sont d'une énergie égale à celle de l'ascète ou de l'ermite, habités qu'ils sont de la rage paranoïaque de représenter et de rendre justice $^{17}$.

Certes, nous dit Bernard-Henri Lévy, « les socialistes représentent peut-être cet ultime quartier de noblesse dans un univers sans souveraineté ${ }^{18}$, certes "le monde serait peut-être meilleur si nous étions encore pieux ${ }^{19}$. Mais leur mensonge n'en est pas moins grand, il n'en est que plus mystificateur. «Le christianisme consolait en promettant le paradis » au ciel. Le marxisme console, dit-on, lui aussi en promettant une société heureuse. Le christianisme, armé d'une dialectique du temps et de l'éternité, attribuait à l'histoire, à la misère séculaire des individus et des peuples, toutes les vertus providentielles du rachat et de la résurrection. L'histoire se trouvait ainsi doté d'un

\footnotetext{
14. Idem, p. 30 .

15. Idem, p. 53 .

16. Idem, p. 30 .

17. Idem, cf. pp. 186 et 196.

18. Idem, p. 196.

19. Idem, p. 196.
} 
sens. Le marxisme, dit-on, ne fait pas autrement puisque par le biais de la dialectique des luttes de classes, doublée d'une croyance dans l'irréversibilité du progrès, il a lui aussi ses martyres, l'histoire devenant dans le creuset de la douleur, " le lieu du bien, l'accoucheuse du mieux, le chemin sûr et béni de l'obligée révolution ${ }^{20}$.

La différence réside sans doute en ceci, nous dit-on, que l'imposture du marxisme est visible : elle se constate dans l'échec temporel du socialisme, dans le totalitarisme, dans les affres du fascisme stalinien et dans ses irréductibles séquelles. "L'imposture du socialisme a commencé quand on a vu l'antichambre du bonheur et la fin de la diaspora ${ }^{21}$, quand on y a vu «la clé d'or qui ouvre les portes du paradis $»^{22}$.

Or, le nouveau philosophe cherche à nous en convaincre, l'histoire n'a pas de sens, l'histoire est un bourbier, il n'y avait pas à l'origine de ver dans le fruit de l'histoire que l'histoire se serait chargée ensuite d'extirper, pas plus qu'il n'y avait à l'origine une nature saine "qu'un péché tard venu ${ }^{23}$ aurait viciée. L'histoire est un fruit verreux, du commencement à la fin, et «le péché c'est Marx ${ }^{24}$ qui a cru pouvoir en aviser autrement. Pour le nouveau philosophe, il n'y a pas d'histoire qui fait sens ; " le passé n'est qu'un labyrinthe de boue insensé et stochastique $"{ }^{25}$, l'historien un vendeur d'illusions et de songes creux. Que répondre à cela ?

D'abord que le nouveau philosophe est sans doute inconséquent puisque octroyer à l'histoire la fatalité d'un cycle infernal et insensé qui, de fois en fois, reproduit sous des modalités différentes le mal radical du lien social, c'est déjà attribuer à l'histoire un sens : le non-sens, le chaos de l'origine se déployant par spirales dans l'histoire est signifiant, mais il l'est sous le mode du pessimisme. C'est le nouveau philosophe de l'histoire qui en détient le devis lui qui a la prétention, comme tout autre

\footnotetext{
20. Idem. p. 87.

21. Idem, p. 96.

22. Idem, p. 96.

23. Idem, cf. p. 181.

24. Idem, p. 181.

25. Idem, cf, p. 64 .
} 
historien, de rendre compte de l'histoire telle qu'elle fut, lui qui a la prétention d'échapper à l'illusion.

Sa prétention c'est aussi de rendre compte de la vénalité des temps présents et futurs. Alléguer une ontologie fataliste des rapports de socialité pour signifier son propre désespoir c'est, à notre sens, une façon toute subtile de s'assurer la maîtrise du temps. Sans doute le nouveau philosophe de l'histoire n'est pas, comme le marxiste qu'il allègue, un horloger guettant dans la concaténation nécessaire et obligée de l'histoire le moment propice et apocalyptique des révolutions prometteuses, mais il n'en est pas moins un témoin imposant à l'histoire la logique de son propre désespoir et prophétisant la noirceur des siècles à venir. Un tel désespoir est à sa manière rassurant puisqu'il estompe lui aussi toute marge d'incertitude.

Faudrait-il penser aussi avec le nouveau philosophe que rien dans l'histoire n'a changé pour le mieux, d'un seul iota, que, pour reprendre le mot de Bernard-Henri Lévy, "demain sera comme aujourd'hui tant qu'il y aura de l'histoire et de la culture ${ }^{26}$ ? Faudrait-il penser qu'entre le travailleur syndiqué de l'Occident contemporain et le prolétariat des taudis du capitalisme sauvage tel qu'il s'est pratiqué dans l'Angleterre coloniale au XIX ${ }^{\mathrm{e}}$ siècle ou tel qu'il se pratique encore dans la phase de l'impérialisme, en Rhodésie ou en Afrique du Sud, rien n'a changé ? Qu'entre le servage médiéval et le salariat moderne tout est du pareil au même, qu'entre l'esclavagisme gréco-romain ou égyptien et la classe ouvrière des grands centres urbains industriels il n'y a qu'une différence de nuance, que nous retournerions tous volontiers, collectivement et sans grande répugnance au pouvoir absolu de vie ou de mort de la caste patricienne, au travail forcé, au fouet? Faudrait-il penser qu'entre la Chine féodale et la Chine nouvelle, qu'entre l'esclavagisme des plantations de tabac et de canne à sucre et la république socialiste de Cuba, qu'entre la Russie des Tsars et des moujiks affamés et illettrés et celle des Soviets, rien ne s'est passé, qu'il nous serait parfaitement indifférent, mal pour mal, d'en revenir aux techniques primitives d'exploitation des sols et des sous-sols, aux épi-

26. Idem, p. 39. 
démies médiévales, que le développement des sciences est une ânerie ?

Le péché de Marx, s'il en fut un, fut de croire que les révoltes et les révolutions historiques ne furent pas l'expression d'un simple.caprice d'intellectuel optimiste, qu'elles répondirent à une nécessité, qu'il en allait de la survie même des révoltés, et que partout où il y a oppression on ne peut s'attendre à autre chose qu'au renversement des structures totalitaires, que le totalitarisme recule en changeant de forme et que chaque révolution réussie, pour autant qu'elle fut inévitable, a permis au peuple des opprimés d'arracher par bribes, par lambeaux, par morceaux, des droits qui ne lui étaient pas reconnus et que le procès de libération des peuples n'aura de cesse de se poursuivre tant qu'il y aura oppression. Tout cela ne signifie pas pourtant l'abrogation de la violence, la parade vers le « happy end " historique, car jusqu'à nouvel ordre, et peut-être encore pour longtemps, la libération passera par le chemin étroit de la rébellion et de la violence, toujours impur pour le philosophe qui rêve d'une paix sociale spontanée et qui abhorre la politique. Le philosophe, s'il veut la paix, s'il ne peut vouloir que la paix, doit aussi être concerné par la violence, celle qui vient du haut et celle qui vient du bas. Libération et barbarie se côtoient et se croisent et on ne peut penser qu'il puisse en aller autrement tant et aussi longtemps que se reproduiront les grandes sources de la barbarie : l'appropriation par les minorités, pour les minorités, de l'argent, de la nature et du pouvoir.

Que doit-on alors reprocher à Marx ? Un optimisme béat qu'il n'a jamais eu ? Un parti pris contemplatif pour la société heureuse que son travail inlassable et quotidien de militant n'a jamais sanctionné ? Une vision mécaniste et eschatologique de l'histoire que lui a prêtée la bourgeoisie libérale et une certaine élite chrétienne pour mieux produire ses propres effets performatifs et qu'ont en partie entérinée les soi-disant marxistes économistes dépourvus de volonté politique ? Ou doit-on lui reprocher encore que l'idéal du socialisme n'ait pas enfanté un socialisme idéal, qu'entre le projet socialiste de Marx et les misères du socialisme historique se soient interposées les forces même de l'histoire sur lesquelles Marx n'a jamais prétendu exercer sa propre tutelle, lui qui savait mieux que tout autre que ce ne 
sont pas les idées, si louables soient-elles, qui font l'histoire mais les luttes générées par la nécessité de vivre contre la tyrannie des possédants?

Qu'on y regarde de près : Marx n'a jamais jugé utile de brosser le tableau quotidien de cette société platement heureuse dont on veut à tout prix lui attribuer la paternité. Aussi la Commune de Paris est apparue à Marx comme un exemple de démocratie populaire mais la Commune de Paris, aux yeux de Marx, n'avait rien pour autant d'un paradis terrestre. De même, pour Marx, le prolétariat industriel n'avait rien d'une classe miracle, lucide et homogène, marchant d'un même pas. Encore lui fallait-il dans sa propre division la volonté politique de s'affranchir du plein pouvoir de la bourgeoisie et la convergence des conditions socio-politico-économiques de crise qui l'inciteraient à y accéder. Et c'est, je crois, cette volonté politique des socialistes que l'on prend pour de la prophétie scientifique ${ }^{27}$, alors qu'elle ne fait qu'exprimer pour Marx qu'entre la société barbare et la société heureuse il y a la société possible.

Peut-être le nouveau philosophe lit-il mieux l'avenir dans la transparence de sa boule de cristal, lui qui s'est délesté de toute incertitude, lui qui sait d'avance que la bourgeoisie a ceci de particulier, contrairement à toute autre classe dominante antérieure, qu'elle est increvable, qu'elle peut récupérer toute dissidence, qu'elle peut tourner tout conflit à son propre profit, que toute crise lui est salutaire. Lui qui sait mieux que tout autre que le socialisme n'est et ne peut être que l'ultime procès d'universalisation du pouvoir et de la répression, l'État s'appropriant plus impitoyablement encore au nom du bien collectif le contrôle tentaculaire dont se gorge la bourgeoisie sous le couvert de la privatisation des libertés.

Sans doute on ne peut qu'être obligeant à l'endroit des nouveaux philosophes de nous rappeler intempestivement qu'à l'Est comme à l'Ouest rien ne va pour le mieux dans le meilleur des mondes. Notre siècle, pour peu que l'on y soit attentif, est secoué, lui aussi, brutalement par les spasmes de la barbarie. Pouvoirs et micro-pouvoirs se démultiplient au rythme de crois-

27. Idem, cf. p. 100 . 
sance des ramifications et des sophistications des appareils de contrôle et de gestion bureaucratiques. Les idéologies « démocratiques » officielles de droite et de gauche sont en crise et la réalité ne cesse de sécréter les démentis qui crèvent l'illusion confortable de la sacro-sainte vertu de leurs fantasmes rédempteurs. Indéniablement encore, la mondialisation des rapports de force entre les superpuissances socialiste et capitaliste exaspère les dogmatismes, durcit les positions et pousse sur le règlement violent des antagonismes nationaux de classe, sur les revendications à l'habitat, au travail, à l'éducation, aux denrées alimentaires, aux libertés civiles dans l'ensemble des communautés qui peuplent la planète. Un pas de plus, en ce siècle de militarisation, et il ne faudrait pas se surprendre que le règlement optimal de notre barbarie ne se retrouve finalement dans un « apocalyptique " chassé-croisé de missiles nucléairłs à têtes simples ou mirvées. Soit ! Il est donc plus qu'utile, sur l'invitation des nouveaux philosophes, que l'on se départisse de nos petits dogmes et de nos petites obsessions de droite ou de gauche ne fût-ce que pour mieux mesurer le spectre entier des vices effectifs des institutions et des systèmes politico-économiques que l'on privilégie abstraitement. On ne risquera jamais de mourir d'une surdose d'esprit critique.

Mais qu'on le veuille ou non, il est tout aussi vrai que l'organisation matérielle et culturelle des collectivités repose sur la médiation politique des rapports sociaux et qu'en cette matière on ne peut éviter aujourd'hui l'alternative de choisir " philosophiquement " et "politiquement " entre le socialisme et le capitalisme, entre l'appropriation privée et l'appropriation collective des ressources naturelles et techniques, entre la socialisation et la privatisation de la gestion économique et politique des institutions sociales, quelle que soit par ailleurs la pertinence ou l'impertinence des « modèles " historiques sur lesquels, en certaines petites églises, on ne cesse de rabattre ou d'asseoir sa croyance. L'abstention ne change rien au cours de la réalité et tout repli sur une éthique provisoire, apolitique, ne peut que donner lieu à un nouveau sacerdoce : celui du " nouvel » ermite protestant des entrailles de sa philosophique retraite de l'imperfection ontologique du monde. Qu'on se le dise : le socialisme totalitaire et le capitalisme tentaculaire ne sont pas au bout de 
leurs misères et de leurs confrontations. Et l'on n'aura de répit dans les décennies à venir d'en voir éclater les formes. Aucun " système ", quel qu'il soit, ne subsiste sans n'avoir à ingurgiter en retour les turbulences qu'il engendre et sans n'être contraint un jour ou l'autre de se refaire la peau, si tant est que les « masses » et les « individus », loin d'être de simples doubles patentés du Prince, ont aussi leurs désirs et leurs mouvements de colère. On n'accédera sans doute jamais aux plaisirs rêvés d'une terre paradisiaque ni à l'abrogation des pouvoirs et des micro-pouvoirs, faut-il insister, mais il est tout aussi vrai encore que l'on n'en finira jamais d'interpeller l'essence énigmatique d'une démocratie à produire dans les faits.

Qu'ils nous disent donc ces nouveaux philosophes, déprimés par la malédiction pérennelle qui frappe le pouvoir, et par l'imposture de toute politique, comment ils satisferont eux aux besoins de survie quotidienne et de justice du peuple des exploités, des démunis et des "muselés ", si ce n'est précisément en acceptant de se «salir les mains" dans les vicissitudes de la révolte et de la politique. Qu'ils nous disent eux qui se targuent et je cite - " de ne jamais plus se mettre au service des révoltés qui n'ont que faire de leurs lumières, de ne jamais plus militer, exilés pour longtemps de ce qu'on appelle la politique, de ne jamais plus porter les rêves des hommes dans leurs bras ${ }^{28}$ qu'ils nous disent comment du tréfonds de leur " morale provisoire $"{ }^{29}$ et de leur virginité philosophique ils feront passer dans l'histoire leur propre morale qui ambitionne - et je cite encore - « contre toute espérance, de penser jusqu'au bout, de penser sans la croire, l'impossible idée d'un monde soustrait à la Maîtrise ${ }^{30}$. On veut, dit-on, changer l'homme en ce qu'il a de plus profond, mais on ne s'interroge guère sur la difficulté des moyens pour y parvenir. On veut, nous dit Bernard-Henri Lévy, "veiller à ce que notre monde, impossible à reconstruire, ne se défasse point ${ }^{31}$ mais on a déjà trop bien conclu que le monde est un désastre.

28. Idem, pp. 223-224.

29. Idem, p. 96.

30. Idem, p. 224.

31. Idem, p. 223. 
Mais à quoi bon insister puisque pour le nouveau philosophe la révolte et le pouvoir ne sont ni le lieu ni la place qui reviennent au philosophe. À quoi bon insister puisque pour le nouveau philosophe il n'y a au fond ni dominateurs, ni dominés, puisque le mal est bien ailleurs, dans la socialité même de l'homme.

Que répondre encore? Que pour Marx la socialité n'est pas une malédiction mais au contraire la condition même de la survie et de l'évolution de l'espèce. Que le pouvoir n'est pas en soi une fatalité puisque ce qui importe c'est de savoir chercher sans vacances qui peut l'exercer, au nom de qui, pour l'intérêt de qui, et par quels moyens.

Qu'ont donc les nouveaux philosophes à nous dire outre l'amputation et la simplification délirante qu'ils opèrent sur la pensée de Marx et outre l'ontologie du désespoir qu'ils cherchent à nous inoculer ? Qu'il existe des marxistes qui citent Marx comme on cite une Bible ? C'est juste. Qu'il existe des marxistes qui surnagent dans le dogmatisme d'une science pétrifiée où tout est toujours déjà expliqué ? Il en existe. Que le stalinisme, que le Goulag, que les camps concentrationnaires, que le dirigisme tyrannique de certains partis communistes ont discrédité l'esprit du socialisme? Aucun socialiste encore pourvu d'une once de lucidité ne saurait renier que les vicissitudes de la politique ne peuvent tout excuser ni tout expliquer. Et c'est sans doute encore ici qu'il faut savoir entendre parler les nouveaux philosophes.

Mais il faut aussi savoir se rappeler que, pour le nouveau philosophe, tout est perdu d'avance, qu'il n'y a pas d'alternative à la barbatie, et que ce pessimisme radical équivaut peut-être, à la limite, à sanctionner la nécessité inéluctable de la barbarie. Et s'il en est ainsi, c'est que le nouveau philosophe libertaire entretient lui-même, à son insu, une idée telle du bonheur et de la société heureuse (qu'il ne saurait nommer) qu'il ne peut que désespérer lorsqu'il s'agit de mettre le pied dans la porte de l'histoire politique.

Car pour lui, la politique est pour les damnés de ce monde, assoiffés de stratégies et de manœuvres machiavéliques. Quant à la philosophie, réduite à sa dimension solipsiste, elle est la 
chasse gardée des âmes pures, souffrantes et hérö̈ques et le mariage de l'une et de l'autre ne peut engendrer qu'une progéniture monstrueuse. Curieuse division du travail que cette séparation franche et radicale de la Politique et de l'Éthique ${ }^{32}$ qui devient pour le nouveau philosophe la seule planche de salut, dût-il convenir, comme il le fait, que l'éthique reste malgré tout impuissante et que la politique conduit irrémédiablement au totalitarisme. Ultime paradoxe d'une vision du monde qui se repaît de paradoxes.

Car si le nouveau philosophe dénonce la barbarie du Capital et le totalitarisme socialiste, s'il les stigmatise sans y apporter les nuances qu'il faudrait, il ne craint pas pourtant de dire que - et je cite :

Il n'y a pas de domination, que l'oppression n'existe pas, que le prince n'a pas de trône et pas de lieu assignable, que nous sommes des opprimés sans oppresseurs qui nous dominent ${ }^{33}$.

Car le mal est toujours ailleurs. Il siège au cœur même de la socialité originelle des hommes, condamnés à vivre ensemble. N'y aurait-il sur cette terre que deux hommes, ce serait déjà trop. Car il n'en faut pas plus pour voir surgir la maîtrise.

Département de philosophie

Université du Québec à Trois-Rivières

32. Idem, cf. p. 84

33. Idem, pp. 28-29. 